\title{
3D Reconstruction of the Surface Using a Standard Camera
}

\author{
Dalibor Martišek \\ Faculty of Mechanical Engineering, Institute of Materials Science and Engineering, NETME Center, Brno University of Technology, \\ Technická 2, 61669 Brno, Czech Republic \\ Correspondence should be addressed to Dalibor Martišek; martisek@fme.vutbr.cz
}

Received 5 August 2017; Accepted 10 October 2017; Published 29 November 2017

Academic Editor: Yakov Strelniker

Copyright (C) 2017 Dalibor Martišek. This is an open access article distributed under the Creative Commons Attribution License, which permits unrestricted use, distribution, and reproduction in any medium, provided the original work is properly cited.

\begin{abstract}
This paper deals with 3D reconstructions of series of partially focussed images. Some of these methods are known in case of images which were acquired in small field of view (by confocal microscope or CCD camera, e.g.). In this case, recorded images do not differ in any geometrical transformation from each other. In case of larger samples (oversized for microscope or CCD camera), it is necessary to use wider viewing field (standard cameras, e.g.), and taken images primarily differ in scaling but may also differ in shifting and rotation too. These images cannot be used for reconstruction directly; they must be registered; that is, we must determine all transformations which the images differ and eliminate their effects. There are several ways to do this. This paper deals with the registration based on phase correlation. After this registration, it is necessary to identify the sharp parts and to compose a 2D and 3D model. Present methods are very sensitive to noise and their results are not satisfactory in many cases. We introduce a new method for 3D reconstruction which is significantly better.
\end{abstract}

\section{Introduction}

The three-dimensional reconstruction of general surfaces plays an important role in many branches; for example, the morphological analysis of fracture surfaces reveals information on mechanical properties of natural or construction materials.

There are more techniques capable of producing digital three-dimensional (3D) replicas of solid surfaces. Mechanical engineers can use contacting electronic profilometers to determine digital two-dimensional (2D) profiles that can be combined into 3D surface profiles; see [1,2], for example. The contacting mode of atomic force microscopes actually belongs to this mechanical category [3]. Besides the mechanical tools, there exist different optical devices [2], light section microscopy $[4,5]$, coherence scanning interferometry [6], speckle metrology [7], stereo projection [8], photogrammetry [9], and various kinds of light profilometry [10], to mention some of them.

3D laser scanning techniques are the next possibilities of how to obtain the 3D data. These techniques have also been tested in some rock engineering projects, such as $3 \mathrm{D}$ digital fracture mapping [11-13].
However, these devices are not of universal use. Each of them has technical limits $[14,15]$. For example, very rough surfaces can hardly be measured by atomic force microscopes, which work in the nanoregions. On the other hand, plane surfaces with microscopically small irregularities may be measured, for example, by the microscopic sectional technique within the so-called confocal microscopes [16-19]. However, confocal microscope is often not suitable for technical purposes due to the small size of the visual field (maximal visual field is approximately $2 \mathrm{~cm}[5,20-$ 22]).

In this paper, how to perform the $3 \mathrm{D}$ reconstruction of larger surfaces using a standard camera will be shown.

\section{Materials and Methods}

2.1. Equipment. In technical practice the confocal microscope is used as a standard tool for imaging microscopic three-dimensional surfaces. The depth of the optical field of the microscope is very small and its advanced hardware is capable of removing nonsharp points from the images. The points of the object close to the focal plane are visible as sharp points. The parts lying above or beneath the focal plane are 


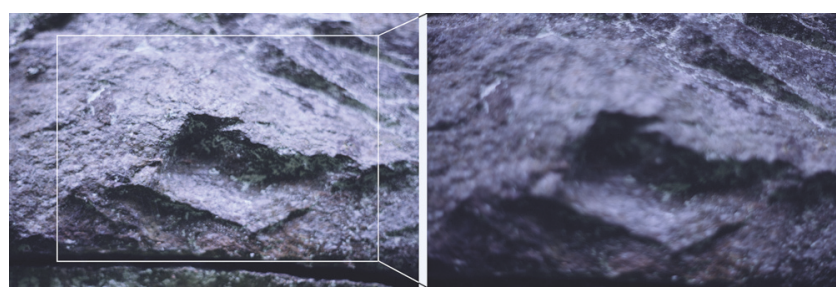

FIGURE 1: Different scaling and different sharp and nonsharp regions in images acquired by classic camera placed in different distances from the 3D relief, the first and the fifteenth images from the series of fifteen images of blue marble. Frame size $10 \times 7.5 \mathrm{~cm}$. Locality Nedvědice, Czech Republic, photo Pavel Štarha.

invisible and represented as black regions. To create a $2 \mathrm{D}$ and $3 \mathrm{D}$ reconstruction, it is necessary to obtain a series of images of the same object, each of them with different focusing, and each point of the object focussed in one of the images (in the ideal case). The sharp parts are identified and composed of a 2D and 3D model.

However, the confocal microscope is often not suitable for technical purposes due to the small size of the visual field (maximal visual field is approximately $2 \mathrm{~cm}[5,20-22]$ ). Nevertheless, the same principle may be used even in the case of a CCD camera, classical microscope, or camera. For nondestructive scanning, the camera must be mounted on a stand which enables a movement in the direction approximately orthogonal to the surface with a controlled step.

The difference between confocal microscope and standard camera concerns nonsharp regions that are displayed by classic camera, whereas they are missing in the case of a confocal microscope. However, the sharp and blurred areas can be detected by software. The next dissimilarity lies in a central projection which caused different scaling of partial images in the image series (see Figure 1). Different image scaling (including possibly shift and rotation) may be also quantified and corrected. This problem has not been solved in available sources.

2.2. Present Methods. Probably the first attempts to carry out nonconfocal reconstructions come from the seventies and eighties of the last century [23-28]. Blurred areas detectors (or so-called focusing criteria) are based on various principles. Present methods work in three steps.

The First Step. Three-dimensional matrix $\left\{C_{i j k}\right\}$ is stated. Its element $C_{i j k}$ determines statistical range, variance, or standard Fourier transform of certain neighborhood of the pixel $[i ; j]$ in $k$ th image in the series of $n$ images [29].

The Second Step. Maxima $\max _{k}\left\{C_{i j k}\right\}$ in the columns $\left\{C_{i j 1}\right.$; $\left.C_{i j 2} ; \ldots ; C_{i j n}\right\}$ are found. Height $h_{i j}$ is assigned to the pixel $[i ; j]$ if and only if the maximum is detected on the $h$ th image. In this way, a stair-approximation is obtained.

The Third Step. The stair-approximation from the second step is obviously refined by various interpolation of detector values adjacent with these maxima, that is, values $C_{i ; j ; h-1}$;
$C_{i ; j ; h+1}$ : inverse proportionality $[29,30]$, parabolic fits [31-33], or Gaussian fits [34].

In [35], it is stated: "We have verified that there are only small differences between the three-point Gaussian and the three-point parabolic approximations." However, this statement does not indicate an accuracy of the reconstruction. As shown below, this fact can only mean that both reconstructions are roughly equally bad.

A noise is the fundamental problem of the second and the third step. This problem was solved by several ways: varying size of computational pixel windows [33, 36], averaging multiple snapshots taken at each vertical position [26], or input data averaging [37]. However, these methods are able to decrease a noise but the noise cannot be zeroed. Therefore, the maxima computed in the second step need not indicate the height correctly; moreover, any interpolation (the third step) is not useable for noise data from point of view of numerical mathematics.

Except for these inaccuracies, methods cited above must assume the same scale of all images in the series because they are not able to detect image transformations. In the following sections, we will introduce the $3 \mathrm{D}$ reconstruction method which is able to process a series of noisy images acquired using central projection with variable center, that is, in various scaling (various shift and rotation eventually).

\subsection{The Fourier Transform and Phase Correlation}

2.3.1. Standard Fourier Transform and Inverse Transform. Standard (continuous) Fourier transform of function $f(x)$ : $\mathbb{R} \rightarrow \mathbb{C}$ is function

$$
\mathscr{F}(f)(x)=F(\xi)=\int_{-\infty}^{\infty} f(x) e^{-i x \xi} \mathrm{d} x
$$

(if this integral exists and is finite).

Standard (continuous) Fourier transform of function $f(x ; y): \mathbb{R}^{2} \rightarrow \mathbb{C}$ is function

$$
\mathscr{F}(f)(x ; y)=F(\xi ; \eta)=\iint_{\mathbb{R}^{2}} f(x ; y) e^{-i(x \xi+y \eta)} \mathrm{d} x \mathrm{~d} y
$$

(if this integral exists and is finite).

Inverse (continuous) Fourier transform of function $G(\xi)$ : $\mathbb{C} \rightarrow \mathbb{C}$ is function

$$
\mathscr{F}^{-1}(G)(\xi)=g(x)=\frac{1}{2 \pi} \int_{-\infty}^{\infty} G(\xi) e^{i x \xi} \mathrm{d} \xi
$$

(if this integral exists and is finite).

Inverse (continuous) Fourier transform of function $G(\xi ; \eta): \mathbb{C}^{2} \rightarrow \mathbb{C}$ is function

$$
\begin{aligned}
\mathscr{F}^{-1}(G)(\xi ; \eta) & =g(x ; y) \\
& =\frac{1}{4 \pi^{2}} \iint_{\mathbb{R}^{2}} G(\xi ; \eta) e^{i(x \xi+y \eta)} \mathrm{d} \xi \mathrm{d} \eta
\end{aligned}
$$

(if this integral exists and is finite). 
Standard discrete Fourier transform of function $f(x ; y)$ : $\{0 ; 1 ; \ldots ; N-1\}^{2} \rightarrow \mathbb{C}$ is function

$$
\begin{aligned}
\mathscr{F}(f)(\xi ; \eta) & =F(\xi ; \eta) \\
& =\sum_{x=0}^{N-1} \sum_{y=0}^{N-1} f(x ; y) e^{-(2 \pi i / N)(x \xi+y \eta)} .
\end{aligned}
$$

Function $F$ is also called the Fourier spectrum of function $f$. It is possible to obtain the function $f$ from its Fourier spectrum $F(\xi ; \eta)$ using inverse discrete Fourier transform

$$
\begin{aligned}
\mathscr{F}^{-1}(F)(x ; y) & =f(x ; y) \\
& =\frac{1}{N^{2}} \sum_{\xi=0}^{N-1} \sum_{\eta=0}^{N-1} F(\xi ; \eta) e^{(2 \pi i / N)(x \xi+y \eta)}
\end{aligned}
$$

(see [38] for proof). Function $A(\xi ; \eta)=|F(\xi ; \eta)|$ is called amplitude spectrum of $f(x ; y)$.

2.3.2. $\delta$-Distribution. One-dimensional $\delta$-distribution $\delta(x)$ is a limit of a sequence of functions $\delta_{n}(x) ; n \in \mathbb{N}$ such that

$$
\begin{aligned}
& \text { (a) } \lim _{n \rightarrow \infty} \int_{-\infty}^{\infty} \delta_{n}(x) \mathrm{d} x=1 ; \\
& \text { (b) } \lim _{n \rightarrow \infty} \frac{\delta_{n}\left(x_{0}\right)}{\lim _{x \rightarrow 0} \delta_{n}(x)}=0 ; \quad x_{0} \in \mathbb{R}-\{0\} .
\end{aligned}
$$

Two-dimensional $\delta$-distribution $\delta(x ; y)$ is a limit of a sequence of functions $\delta_{n}(x ; y) ; n \in \mathbb{N}$ such that

$$
\begin{aligned}
\lim _{n \rightarrow \infty} \int_{-\infty}^{\infty} \int_{-\infty}^{\infty} \delta_{n}(x ; y) \mathrm{d} x \mathrm{~d} y & =1 ; \\
\lim _{n \rightarrow \infty} \frac{\delta_{n}\left(x_{0} ; y_{0}\right)}{\lim _{(x ; y) \rightarrow(0 ; 0)} \delta_{n}(x ; y)} & =0 ; \\
& \left(x_{0} ; y_{0}\right) \in \mathbb{R}^{2}-\{(0 ; 0)\} .
\end{aligned}
$$

Example 1. One of the well-known examples (which we demonstrate in $1 \mathrm{D}$ for simplicity) is the series of expanding rectangular signals $\delta_{n}^{*}(\xi)$ with constant unitary intensity on $(-n ; n) ; n \in \mathbb{N}$ and zeroed elsewhere. Inverse Fourier transform gives

$$
\begin{aligned}
\mathscr{F}^{-1}\left(\delta_{n}^{*}\right)(\xi) & =\delta_{n}(x)=\frac{1}{2 \pi} \int_{-\infty}^{\infty} e^{i x \xi} \mathrm{d} \xi \\
& =\frac{1}{2 \pi} \int_{-n}^{n} e^{i x \xi} \mathrm{d} \xi=\frac{1}{2 \pi}\left[\frac{e^{i x \xi}}{i x}\right]_{\xi=-n}^{n} \\
& =\frac{e^{i x n}-e^{-i x n}}{2 \pi i x}=\frac{\sin n x}{\pi x} .
\end{aligned}
$$

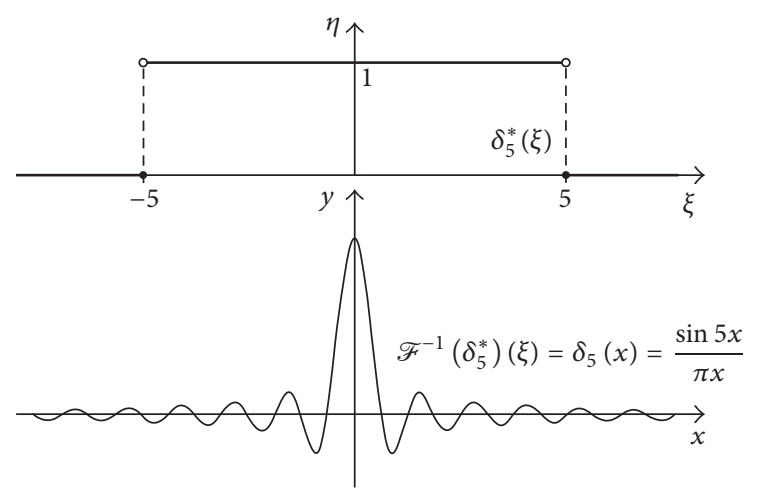

FIGURE 2: Fourier transforms of the fifth member of the series of expanding rectangular signals. The series converges to $\delta$ distribution.

Because $\int_{-\infty}^{\infty}((\sin n x) / \pi x) \mathrm{d} x=1$ for each $n \in \mathbb{N}$ (see [36] for proof), condition (a) is fulfilled. Because $\lim _{x \rightarrow 0}((\sin n x) /$ $\pi x)=(n / \pi) \lim _{x \rightarrow 0}((\sin n x) / n x)=n / \pi$ for each $n \in \mathbb{N}$, there is

$$
\lim _{n \rightarrow \infty} \frac{\delta_{n}\left(x_{0}\right)}{\lim _{x \rightarrow 0} \delta_{n}(x)}=\lim _{n \rightarrow \infty} \frac{\sin n x_{0}}{n x_{0}}=0
$$

for each $x_{0} \neq 0$.

It means that also condition (b) holds and limit $\delta(x)=$ $\lim _{n \rightarrow \infty} \delta_{n}(x)$ is the (one-dimensional) $\delta$-distribution. Expanded unitary signal and its Fourier transform are illustrated in Figure 2.

2.3.3. Phase Correlation. For image processing, it is necessary to transform the images so that the studied structures are at the same position in all the images. This is the task of image registration, to find the transformation. In some applications we assume that images were shifted only; in others we allow shift, rotation, and scale change (i.e., similarity), general linear transformation, or even general transformations. The methods used for registration depend on the expected transformation and on the structures in the image. Some methods use corresponding structures or points in the images and then find a global transformation using the measurements of positions of the structures or points [39-41]. These methods require these structures to be clearly visible. Other methods are based on correlation and work with the image as a whole. The phase correlation proved to be a powerful tool (not only) for registration of particular focussed images. For functions $f_{1} ; f_{2}$, it is defined as

$$
P_{f_{1} ; f_{2}}(x ; y)=\mathscr{F}^{-1}\left\{\frac{F_{1}(\xi ; \eta) \cdot \bar{F}_{2}(\xi ; \eta)}{\left|F_{1}(\xi ; \eta)\right| \cdot\left|F_{2}(\xi ; \eta)\right|}\right\}
$$

and its modification as

$$
\begin{array}{r}
P_{f_{1} ; f_{2} ; p ; q}(x ; y)=\mathscr{F}^{-1}\{H(\xi ; \eta) \\
\left.\cdot \frac{F_{1}(\xi ; \eta) \cdot \bar{F}_{2}(\xi ; \eta)}{\left(\left|F_{1}(\xi ; \eta)\right|+p\right) \cdot\left(\left|F_{2}(\xi ; \eta)\right|+q\right)}\right\},
\end{array}
$$


where strip means complex conjugation and $H(\xi ; \eta)$ is a bounded real function such that $H(\xi ; \eta)=H(-\xi ;-\eta)$ and $p ; q>0$ are arbitrary constants. It can be proved that for real functions $f_{1} ; f_{2}$ the phase-correlation function is real [42]. This is of great value, since it enables us to search for extremes of the phase-correlation function.

\subsection{Image Transformation}

Identical Images. Let $F$ be the infinity periodic expansion of an image. Denote $a+b i$ as its value $F(\xi ; \eta)$ in the point $(\xi ; \eta)$, $a+b i \neq 0$. It is evident that the value of the phase correlation of the $F$ with itself is

$$
\begin{aligned}
\frac{F(\xi ; \eta) \cdot \bar{F}(\xi ; \eta)}{|F(\xi ; \eta) \cdot F(\xi ; \eta)|} & =\frac{(a+b i)(a-b i)}{|(a+b i)| \cdot|a+b i|}=\frac{a^{2}+b^{2}}{a^{2}+b^{2}} \\
& =1 .
\end{aligned}
$$

According to Example 1 in Section 2.3.2, one has

$$
\begin{aligned}
P_{f ; f}(x ; y) & =\mathscr{F}^{-1}\left\{\frac{F(\xi ; \eta) \cdot \bar{F}(\xi ; \eta)}{|F(\xi ; \eta)| \cdot|F(\xi ; \eta)|}\right\}=\mathscr{F}^{-1}\{1\} \\
& =\delta(x ; y) .
\end{aligned}
$$

It means that the inverse Fourier transform of the convolution of two identical images is the two-dimensional $\delta$-distribution $\delta(x ; y)$.

Shifted Images. If two functions are shifted in arguments, that is, $f_{2}(x ; y)=f_{1}\left(x-x_{0} ; y-y_{0}\right)$, their Fourier transforms are shifted in phase; that is,

$$
F_{2}(\xi ; \eta)=F_{1}(\xi ; \eta) \cdot \exp \left(-i\left(\xi x_{0}+\eta y_{0}\right)\right)
$$

and their phase-correlation function is the $\delta$-distribution shifted in arguments by the opposite shift vector

$$
\begin{aligned}
P_{f_{1} ; f_{2}}(x ; y) & =\mathscr{F}^{-1}\left\{\exp \left(i\left(\xi x_{0}+\eta y_{0}\right)\right)\right\} \\
& =\delta\left(x+x_{0} ; y+y_{0}\right)
\end{aligned}
$$

This is the main idea of phase correlation. The task to find a shift between two images is converted by the phase correlation to the task of finding the only nonzero point in a matrix. If the images are not identical (up to a shift), that is, if the images are not ideal, the phase-correlation function is more complicated, but it still has a global maximum at the coordinates corresponding to the shift vector.

Rotated Images. The phase-correlation function can be also used for estimation of image rotation and rescale. Let $f_{2}$ be function $f_{1}$ rotated and shifted in arguments; that is,

$$
\begin{aligned}
& f_{2}(x ; y) \\
& \quad=f_{1}\left(x \cos \theta-y \sin \theta-x_{0} ; x \sin \theta+y \cos \theta-y_{0}\right) .
\end{aligned}
$$

Their Fourier spectra $F_{1} ; F_{2}$ and amplitude spectra $A_{1} ; A_{2}$ are related as follows:

$$
\begin{aligned}
F_{2}(\xi ; \eta)= & \exp \left(-i\left(\xi x_{0}+\eta y_{0}\right)\right) \\
& \cdot F_{1}(\xi \cos \theta-\eta \sin \theta ; \xi \sin \theta+\eta \cos \theta), \\
A_{2}(\xi ; \eta)= & A_{1}(\xi \cos \theta-\eta \sin \theta ; \xi \sin \theta+\eta \cos \theta)
\end{aligned}
$$

The shift results in a phase shift and the spectra are rotated in the same way as the original functions. A crucial step here is transformation of the amplitude spectra into the polar coordinate system to obtain functions $A_{1}^{p} ; A_{2}^{p}: \mathbb{R}_{0}^{+} \times$ $\langle 0 ; 2 \pi) \rightarrow \mathbb{R}_{0}^{+}$such that $A_{1}^{p}(\rho ; \varphi)=A_{2}^{p}(\rho ; \varphi+\theta)$. The rotation around an unknown center of rotation was transformed to a shift. This shift is estimated with the standard phase correlation (see the previous paragraph) after rotating back by the measured angle; the shift $\left(x_{0} ; y_{0}\right)$ is measured with another computation of the phase correlation.

Scaled Images. Let $f_{2}$ be function $f_{1}$ rotated, shifted, and scaled in arguments; that is,

$$
\begin{gathered}
f_{2}(x ; y)=f_{1}(\alpha(x \cos \theta-y \sin \theta) \\
\left.-x_{0} ; \alpha(x \sin \theta+y \cos \theta)-y_{0}\right) .
\end{gathered}
$$

Their Fourier spectra and amplitude spectra are related as follows:

$$
\begin{aligned}
& F_{2}(\xi ; \eta)=\frac{1}{\alpha^{2}} \exp \left(-i\left(\xi x_{0}+\eta y_{0}\right)\right) \\
& \cdot F_{1}\left(\frac{1}{\alpha}(\xi \cos \theta-\eta \sin \theta) ; \frac{1}{\alpha}(\xi \sin \theta+\eta \cos \theta)\right), \\
& A_{2}(\xi ; \eta)=\frac{1}{\alpha^{2}} \\
& \cdot A_{1}\left(\frac{1}{\alpha}(\xi \cos \theta-\eta \sin \theta) ; \frac{1}{\alpha}(\xi \sin \theta+\eta \cos \theta)\right) .
\end{aligned}
$$

The shift results in a phase shift, and the spectra are rotated in the same way as the originalfunctions and scaled with a reciprocal factor. A crucial step here is transformation of theamplitude spectra into the logarithmic-polar coordinate system

$$
\exp \rho=\sqrt{x^{2}+y^{2}} ; \quad x=\exp \rho \cos \varphi ; \quad y=\exp \rho \sin \varphi
$$

to obtain $A_{1}^{p} ; A_{2}^{p}: \mathbb{R}_{0}^{+} \times\langle 0 ; 2 \pi) \rightarrow \mathbb{R}_{0}^{+}$such that $A_{2}^{1 p}(\rho ; \varphi)=$ $A_{2}^{1 p}(\rho-\ln \alpha ; \varphi+\theta)$.

Both rotation and scale change were transformed to a shift. The unknown angle $\theta$ and unknown factor $\alpha$ can be estimated by means of the phase correlation applied on the amplitude spectra in the logarithmic-polar coordinate system $A_{1}^{1 p} ; A_{2}^{1 p}$. After rotating function $f_{2}$ back by the estimated angle $\theta$ and scaling by factor $\alpha$, the shift vector $\left(x_{0} ; y_{0}\right)$.

\subsection{Proposed Method of Surface Approximation}

2.5.1. Preprocessing: Image Registration. Before 3D profile calculation, it is necessary to identify all geometric 
transformation in the image series and to eliminate them. The images are analyzed as geometrically similar. Generally, similarity is a combination of rotation, scale change, shift, and axial symmetry. Axial symmetry is not possible in our case.

The discrete Fourier transform described above is used for the image registration. It either works with periodic functions or makes them periodic. In general case, an image has not the same values on the edges and by periodizing an image, we obtain a function with jumps at the edges of the original image. These jumps are often the most contrast structures in the function and may lead to incorrect registration. Therefore, it is necessary to remove such edges from the image used for the shift estimation, to smooth them out. This is done by multiplying the image by a suitable function, a so-called window function. Such function must be zero or almost zero at the image edges and one on a large part of the image. We can primarily use the Gaussian window function and the Hanning window function. Let $\sigma \in \mathbb{R}^{+}$be a given number and set

$$
\begin{aligned}
& \mathscr{R}=\langle-a ; a\rangle \times\langle-b ; b\rangle ; \quad a ; b \in \mathbb{R}_{0}^{+}, \\
& \mathscr{C}=\left\{(x ; y) \in \mathbb{R}^{2} \mid x^{2}+y^{2} \leq r\right\} ; \quad r \in \mathbb{R}_{0}^{+} .
\end{aligned}
$$

Let $\rho(X ; \mathcal{S})$ be the distance of point $X=(x ; y)$ from set $\delta$; that is,

$$
\rho(X ; \mathcal{S})=\inf \{d \in \mathbb{R} \mid d=\|X Y\| ; Y \in \mathcal{S}\} .
$$

Functions

$$
\begin{aligned}
g_{\mathrm{GR}}(x ; y) & =e^{-\rho^{2}(X ; \mathscr{R}) / \sigma^{2}} \\
\text { or } g_{\mathrm{GC}}(x ; y) & =e^{-\rho^{2}(X ; \mathscr{C}) / \sigma^{2}}
\end{aligned}
$$

are called rectangular or circular Gaussian window function. Functions

$$
\begin{aligned}
& g_{\mathrm{HR}}(x ; y) \\
& \quad= \begin{cases}\frac{1}{2}+\frac{1}{2} \cos \frac{\pi \rho(X ; \mathscr{R})}{\sigma} & \text { if }(X ; \mathscr{R}) \leq \sigma \\
0 & \text { if }(X ; \mathscr{R})>\sigma\end{cases} \\
& \text { or } g_{\mathrm{HC}}(x ; y) \\
& = \begin{cases}\frac{1}{2}+\frac{1}{2} \cos \frac{\pi \rho(X ; \mathscr{C})}{\sigma} & \text { if }(X ; \mathscr{C}) \leq \sigma \\
0 & \text { if }(X ; \mathscr{C})>\sigma\end{cases}
\end{aligned}
$$

are called rectangular or circular Hanning window function.

Let $\left\{I_{1} ; I_{2} ; \ldots ; I_{n}\right\}$ be the image series to be registered; image $I_{1}$ was acquired by means of the biggest angle of view. This image will be not transformed or (formally) it will be transformed by identity mapping to the image $I_{1}^{*}$. Now we must find transform $I_{2} \rightarrow I_{1}^{*}$ to obtain image $I_{2}^{*}$ which differs from $I_{1}^{*}$ in focussed and blurred parts only. In the same way, transforms $I_{3} \rightarrow I_{2}^{*} ; \ldots ; I_{k} \rightarrow I_{k-1}^{*} ; \ldots ; I_{n} \rightarrow I_{n-1}^{*}$ must be found.

After the multiplying both images $I_{k} ; I_{k-1}^{*}$ by the chosen window function, we determine the rotation angle $\theta_{k}$ and

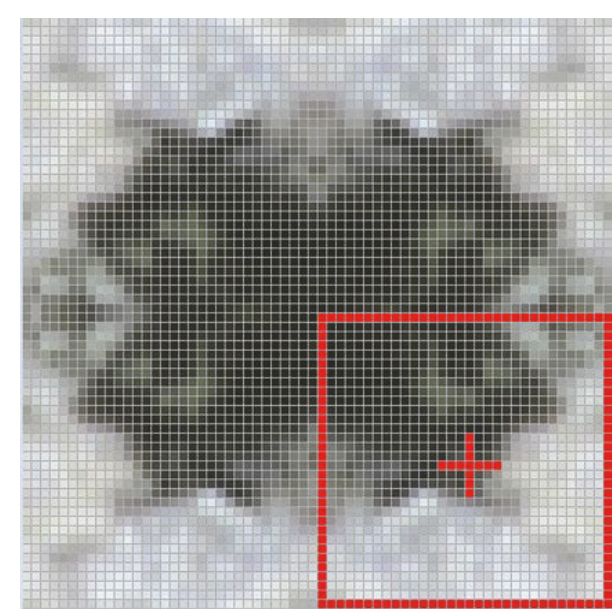

FIGURE 3: Even extension of the $32 \times 32$ neighborhood (framed) of the processing pixel (cross).

scale factor $\alpha_{k}$ by means of the method described in Section 2.4. Then, image $I_{k}$ is rotated by angle $-\theta_{k}$ and scaled by factor $\alpha_{k}^{-1}$ to compensate the rotation and scale change found by the phase correlation, creating image $\overline{I_{k}}$. Only shift and different focussed and blurred parts remain between image $\overline{I_{k}}$ and image $I_{k-1}^{*}$. Now we can apply phase correlation to find the shift $\left(x_{0} ; y_{0}\right)$ and image $\overline{I_{k}}$ is shifted by vector $\left(-x_{0} ;-y_{0}\right)$ to compensate the shift, creating image $I_{k}^{*}$ which differs from $I_{k-1}^{*}$ in focussed and blurred parts only.

2.5.2. The First Step: Matrix of Sharpness Detectors. As was said in Section 2.2, statistical range, variance, or standard Fourier transform of certain neighborhood of the pixel $[i ; j]$ may be used as the sharpness detectors (focusing criteria). The neighborhood of the pixel $[i ; j]$ is a square of $s \times s$ pixels where $s$ is obviously ten to twenty. In case of the standard Fourier transform, algorithm FFT is used. It requires the square with the side $s=2^{n}$; that is, $s=8$ or $s=16$ is used in this case. However, standard Fourier transform suffers from jumps at the edges of the square and it is necessary to use a suitable window function like in Section 2.5.1. Therefore, cosine Fourier transform is preferable. This transform is obtained from the standard Fourier transform which is applied to even extension of the neighborhood to be processed. This extension is illustrated in Figure 3. Due to this extension, there are no jumps at the edges. Sine frequencies in (5) are zeroed and application of any window function is not necessary.

However, low frequencies in amplitude spectrum detect blurred parts of image and very height frequencies are given by noise. Therefore, suitable weight must be assigned to each frequency in sharpness detector calculation. In our software, the following detectors may be used:

$$
\begin{aligned}
{ }_{a} C_{i j k} & =\sum_{m=-s}^{s} \sum_{n=-s}^{s}{ }_{a} c_{i+m ; j+n ; k} \\
& =\sum_{m=-s}^{s} \sum_{n=-s}^{s}(|m|+|n|) \cdot\left|F_{i+m ; j+n ; k}\right|,
\end{aligned}
$$




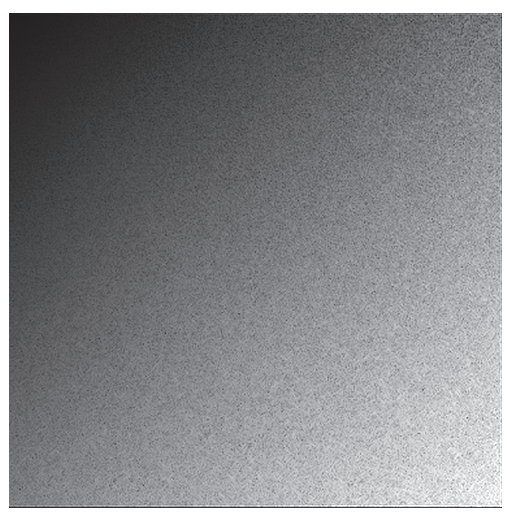

(a)

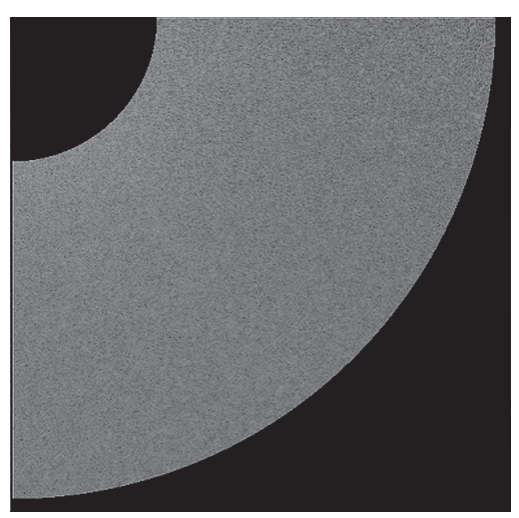

(b)

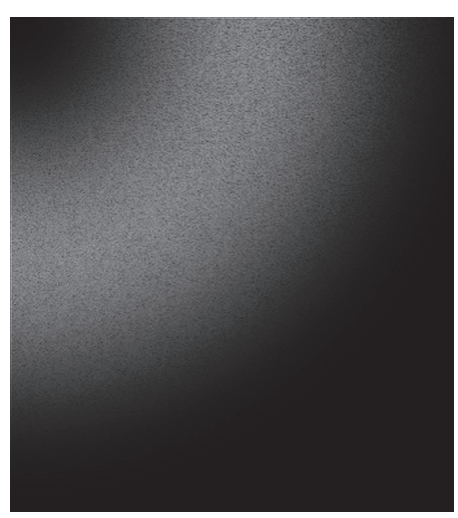

(c)

FIgURE 4: Graphical representation of sharpness detectors ${ }_{a} C(\mathrm{a}) ;{ }_{b} C(\mathrm{~b})$; and ${ }_{c} C(\mathrm{c})$.

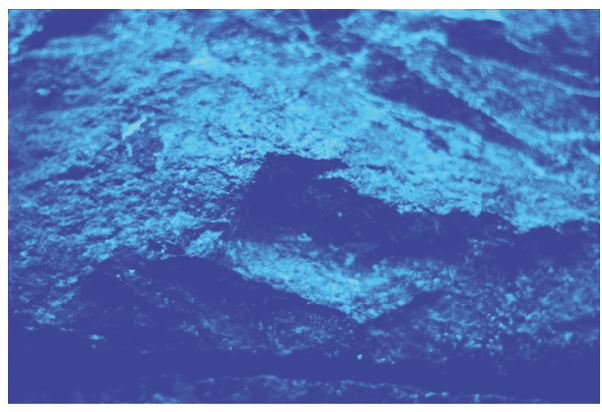

(a)

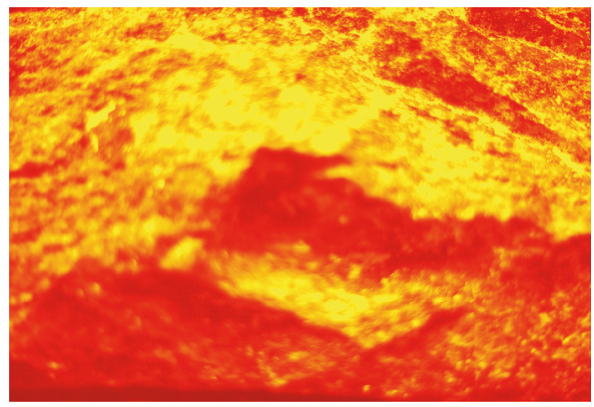

(b)

Figure 5: The first and the fifteenth images from the series of fifteen images of blue marble (see Figure 1) displayed in supplementary pseudocolors. Real surface size $10 \times 7.5 \mathrm{~cm}(\mathrm{~b})$.

$$
\begin{aligned}
{ }_{b} C_{i j k} & =\sum_{m=-s}^{s} \sum_{n=-s}^{s}{ }_{b} c_{i+m ; j+n ; k}=\frac{1}{S\left(\mathscr{A}_{i j}\right)} \sum_{(m ; n) \in \mathscr{A}_{i j}}\left|F_{m n k}\right| \\
{ }_{c} C_{i j k} & =\sum_{m=-s}^{s} \sum_{n=-s}^{s}{ }_{c} c_{i+m ; j+n ; k} \\
& =\sum_{m=-s}^{s} \sum_{n=-s}^{s}\left|F_{i+m ; j+n ; k}\right| \cdot \sin ^{2}\left(\frac{\pi}{s} \sqrt{m^{2}+n^{2}}\right)
\end{aligned}
$$

where $F$ is the cosine spectrum of the pixel neighborhood and $S\left(\mathscr{A}_{i j}\right)$ is volume of the annulus $\mathscr{A}_{i j}$ with the center $(i ; j)$. Elements ${ }_{a} c ;{ }_{b} c ;{ }_{c} c$ which are summed in (26); (27); (28) are illustrated as pixel values in Figure 4. Size of detectors really used in software is $16 \times 16$ or $32 \times 32$ pixels. In Figure 4 , higher resolution is used for better illustration.

2.5.3. The Second Step: Profile Heights Calculation. The main imperfection of current methods is the hypothesis that the profile height in given pixel is exactly determined by values of chosen sharpness detector. This hypothesis implies that these values can be interpolated. However, this conclusion is quite false. We have available the series $\left\{C_{i j k}\right\} ; k=1 ; 2 ; \ldots ; n$ for assessment of the height of pixel $(i ; j)$. This series is not deterministic but it is a random variable. It cannot be interpolated, and it must be processed by statistical method. One of the possibilities is a regression analysis but it would be very complicated. Direct calculation of the expected value is much easier.

For each pixel $(i ; j)$, we can construct theoretically infinitely many probability distribution functions $p_{i j}^{(r)}$ using different exponents $r$ applied to series members $C_{i j k}$ :

$$
p_{i j}^{(r)}(k)=\frac{C_{i j k}^{r}}{\sum_{s=1}^{n} C_{i j s}^{r}} .
$$

Expected values of random variables $P_{i j}^{(r)}$ given by these probability functions estimate the height $h_{i j}^{(r)}$ of surface in its pixel $(i ; j)$ :

$$
h_{i j}^{(r)}=E\left(P_{i j}^{(r)}\right)=\sum_{k=1}^{n} k \cdot p_{i j}^{(r)}(k)=\sum_{k=1}^{n} k \cdot \frac{C_{i j k}^{r}}{\sum_{s=1}^{n} C_{i j s}^{r}} .
$$

\section{Results and Discussion}

3.1. The Preprocessing: Image Registration. In Figure 5, we can see the photos from Figure 1 but they are displayed in so-called supplementary pseudocolors. If these images were completely identical, then arithmetic mean of the bluegreen image on Figure 5(a) and orange image on Figure 5(b) would be "perfectly grey." Arithmetic mean of these images 


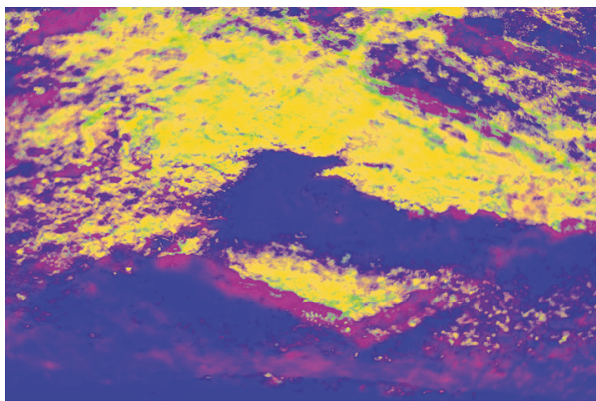

(a)

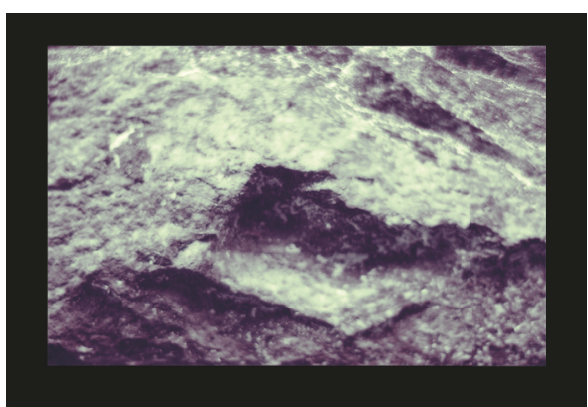

(b)

FIGURE 6: The arithmetic mean of the images from Figure 5: before registration (a) and after registration (b). Real surface size $10 \times 7.5 \mathrm{~cm}(\mathrm{~b})$.

is constructed in Figure 6(a). It is clear that components of this mean are very different, values of the orange image are bigger in yellow parts of the mean, and values of the bluegreen image are bigger in blue-violet parts of the mean.

In Figure 6(b), the same construction after the registration is realized. Very low color saturation of arithmetic mean confirms very good conformity. Of course, arithmetic mean cannot be "perfectly grey" in our case because components of the mean differs in blurred and sharp parts.

In Table 1, indicated and applied transforms in separated images of the blue marble are summarized. All transformations have been detected with subpixel precision; they are listed with a precision of one thousandths pixels. It is obvious that the scaling plays most important role; however, nor shifts are negligible. Rotation angle between the first and the last image is over five arcminutes; it means approximately one pixel on the image periphery (used data resolution is $1600 \times$ 1200 pixels). This transformation is marginal in our case.

3.2. The First Step: Sharpness Detectors. As is clear from (26), (27), and (28) and Figure 4, detector ${ }_{a} C$ increased high frequencies (right bottom corner of squares in Figure 4) too much; it means that it is very noise sensitive. The disadvantages of the detector ${ }_{b} C$ are too sharp cuts which remove low and height frequencies.

Differences between the sharpness detectors stated in Section 2.5.2 are best demonstrated by their maxima. The graphical representation of detector ${ }_{c} C$ (i.e., corresponding stair-approximation of the surface) is shown in Figure 7.

Differences between representations ${ }_{a} C ;{ }_{b} C$; and ${ }_{c} C$ are visually quite miniscule (therefore, ${ }_{a} C$ and ${ }_{b} C$ are not illustrated); however, differences exist. For their quantification, Root Mean Square Error is as follows:

$$
\operatorname{RMSE}\left(h^{(1)} ; h^{(2)}\right)=\sqrt{\frac{1}{W H} \cdot \sum_{i=0}^{W-1} \sum_{j=0}^{H-1}\left(h_{i j}^{(1)}-h_{i j}^{(2)}\right)^{2}} .
$$

Average Deviation is as follows:

$$
\operatorname{AD}\left(h^{(1)} ; h^{(2)}\right)=\frac{1}{W H} \cdot \sum_{i=0}^{W-1} \sum_{j=0}^{H-1}\left|h_{i j}^{(1)}-h_{i j}^{(2)}\right| .
$$

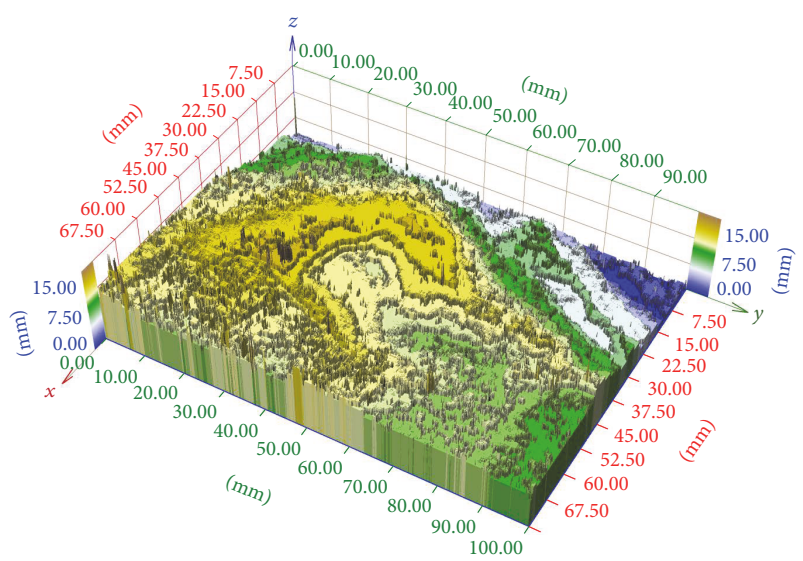

FIgURE 7: The stairs-approximation of the data from Figure 1 constructed by means of the sharpness detector ${ }_{c} C$; see expression (28) and Figure 4(c).

Pearson Correlation Coefficient is as follows:

$$
\begin{aligned}
& \operatorname{PCC}\left(h^{(1)} ; h^{(2)}\right) \\
& =\frac{\sum_{i=0}^{W-1} \sum_{j=1}^{H-1}\left(h_{i j}^{(1)}-\overline{h^{(1)}}\right)\left(h_{i j}^{(2)}-\overline{h^{(2)}}\right)}{\sqrt{\sum_{i=0}^{W-1} \sum_{j=0}^{H-1}\left(h_{i j}^{(1)}-\overline{h^{(1)}}\right)^{2} \sum_{i=0}^{W-1} \sum_{j=0}^{H-1}\left(h_{i j}^{(2)}-\overline{h^{(2)}}\right)^{2}}}
\end{aligned}
$$

and Difference of surface Information Entropy is as follows:

$$
\operatorname{DIE}\left(h^{(1)} ; h^{(2)}\right)=\left|\operatorname{IE}\left(h^{(1)}\right)-\operatorname{IE}\left(h^{(2)}\right)\right|,
$$

where $W ; H$ are width and height of surface domain in pixels; $h_{i j}^{(1)} ; h_{i j}^{(2)}$ are height of first (second) surface in the pixel $(i ; j)$; $\overline{h^{(1)}} ; \overline{h^{(2)}}$ are the average height of the first (second) surface; and

$$
\begin{aligned}
& \operatorname{IE}(h) \\
& =-\sum_{i=1}^{W-1} \sum_{j=1}^{H-1}\left(\frac{h_{i j}}{\sum_{m=1}^{W-1} \sum_{n=1}^{H-1} h_{m n}} \log _{2} \frac{h_{i j}}{\sum_{m=1}^{W-1} \sum_{n=1}^{H-1} h_{m n}}\right)
\end{aligned}
$$

is the Information Entropy of the surface $h$. 
TABLE 1: Parameters of the transforms indicated and applicated to separate images in the series (stated relative to the first image).

\begin{tabular}{|c|c|c|c|c|c|c|c|c|}
\hline \multirow{3}{*}{ Image number } & \multicolumn{4}{|c|}{ Indicated transforms } & \multicolumn{4}{|c|}{ Applicated transforms } \\
\hline & \multirow{2}{*}{ Scale } & \multirow{2}{*}{ Rotation (arcmin.) } & \multicolumn{2}{|c|}{ Shift vector } & \multirow{2}{*}{ Scale } & \multirow{2}{*}{ Rotation (arcmin.) } & \multicolumn{2}{|c|}{ Shift vector } \\
\hline & & & $x$ (pixels) & $y$ (pixels) & & & $x$ (pixels) & $y$ (pixels) \\
\hline (2) & 1.01343 & -0.554 & -1.011 & 1.064 & 0.98675 & 0.554 & 1.011 & -1.064 \\
\hline (3) & 1.02351 & -0.221 & -0.990 & 1.861 & 0.97703 & 0.221 & 0.990 & -1.861 \\
\hline (4) & 1.03796 & -0.061 & -1.869 & 3.027 & 0.96343 & 0.061 & 1.869 & -3.027 \\
\hline (5) & 1.04888 & 0.053 & -2.903 & 3.032 & 0.95340 & -0.053 & 2.903 & -3.032 \\
\hline (6) & 1.06228 & -0.409 & -4.085 & 5.142 & 0.94137 & 0.409 & 4.085 & -5.142 \\
\hline (7) & 1.07055 & -0.140 & -4.947 & 4.987 & 0.93410 & 0.140 & 4.947 & -4.987 \\
\hline (8) & 1.08105 & -0.027 & -4.964 & 5.856 & 0.92503 & 0.027 & 4.964 & -5.856 \\
\hline (9) & 1.09173 & 0.134 & -4.847 & 6.141 & 0.91598 & -0.134 & 4.847 & -6.141 \\
\hline (10) & 1.10340 & 4.652 & -4.988 & 7.003 & 0.90629 & -4.652 & 4.988 & -7.003 \\
\hline (11) & 1.11426 & 5.215 & -5.003 & 7.934 & 0.89746 & -5.215 & 5.003 & -7.934 \\
\hline (12) & 1.12590 & 5.728 & -5.895 & 8.972 & 0.88818 & -5.728 & 5.895 & -8.972 \\
\hline (13) & 1.13784 & 5.278 & -5.907 & 8.872 & 0.87886 & -5.278 & 5.907 & -8.872 \\
\hline (14) & 1.14940 & 5.378 & -5.928 & 8.980 & 0.87002 & -5.378 & 5.928 & -8.980 \\
\hline (15) & 1.16066 & 5.275 & -6.935 & 9.137 & 0.86158 & -5.275 & 6.935 & -9.137 \\
\hline (16) & 1.17297 & 5.324 & -8.036 & 9.095 & 0.85254 & -5.324 & 8.036 & -9.095 \\
\hline
\end{tabular}

TABLE 2: Root Mean Square Error (RMSE) and Average Deviation (AD) in millimeters, Pearson Correlation Coefficient (PCC), and Difference of surface Information Entropy (DIE) (dimensionless quantities) for separate pairs of stairs-approximations ${ }_{a} C,{ }_{b} C$, and ${ }_{c} C$.

\begin{tabular}{lccccccc}
\hline PCC (DL) & \multicolumn{3}{c}{ RMSE $(\mathrm{mm})$} & DIE (DL) & \multicolumn{2}{c}{$\mathrm{AD}(\mathrm{mm})$} \\
& ${ }_{a} C$ & ${ }_{b} C$ & ${ }_{C} C$ & ${ }_{a} C$ & ${ }_{b} C$ \\
\hline${ }_{a} C$ & $\backslash$ & 0.51228 & 0.69630 & ${ }_{a} C$ & $\backslash$ & 0.15258 \\
${ }_{b} C$ & 0.99320 & $\backslash$ & 0.56214 & ${ }_{b} C$ & 0.00505 & $\backslash$ \\
${ }_{c} C$ & 0.98740 & 0.99158 & $\backslash$ & ${ }_{c} C$ & 0.00812 & 0.00307 \\
\hline
\end{tabular}

TABle 3: Root Mean Square Error (RMSE), Average Deviation (AD), Pearson Correlation Coefficient (PCC), and Difference of surface Information Entropy (DIE) for separate pairs of parabolic interpolation approximations ${ }_{a} C,{ }_{b} C$, and ${ }_{c} C$.

\begin{tabular}{lccccccc}
\hline PCC (DL) & \multicolumn{3}{c}{ RMSE $(\mathrm{mm})$} & DIE (DL) & \multicolumn{2}{c}{$\mathrm{AD}(\mathrm{mm})$} \\
& ${ }_{a} C$ & ${ }_{b} C$ & ${ }_{c} C$ & ${ }_{a} C$ & ${ }_{b} C$ \\
\hline${ }_{a} C$ & $\backslash$ & 0.27750 & 0.38644 & ${ }_{a} C$ & $\backslash$ & 0.15066 \\
${ }_{b} C$ & 0.99850 & $\backslash$ & 0.24776 & ${ }_{b} C$ & 0.00464 & $\backslash$ \\
${ }_{c} C$ & 0.99627 & 0.99810 & $\backslash$ & ${ }_{c} C$ & 0.00714 & 0.00250 \\
\hline
\end{tabular}

Values of these characteristics are summarized in Table 2. Note that it is RMSE $=\mathrm{AD}=\mathrm{DIE}=0 ; \mathrm{PCC}=1$ for a pair of identical surfaces.

Similar summary is made in Table 3 for the present reconstruction methods: parabolic interpolation of the stairapproximations ${ }_{a} C,{ }_{b} C$, and ${ }_{c} C$. In Table 4, there are summarized RMSE, AD, PCC, and DIE for parabolic and Gaussian interpolation of the same stair-approximation ${ }_{c} C$ and proposed method of the expected values of columns $\left\{{ }_{c} C_{i j k}\right\} ; k=1 ; 2 ; \ldots ; n$.
As was already stated in [35], only small differences are between parabolic and Gaussian interpolation. Really, values of RMSE, AD, and DIE for parabolic and Gaussian interpolation are approximately thousand times smaller than for interpolation and proposed method. Correlation between interpolations is stated even as one in Table 4. Therefore, it might seem that interpolations are much better than expected value estimation but the opposite is true. Small differences between these methods mean only that these reconstructions are roughly equally bad as shown in Figures 8 and 9. 
TABle 4: Root Mean Square Error (RMSE), Average Deviation (AD), Pearson Correlation Coefficient (PCC), and Difference of surface Information Entropy (DIE) for separate pairs of parabolic interpolation, Gaussian interpolation, and the proposed method applied to the same matrix ${ }_{c} C$. The expected values of columns $\left\{{ }_{c} C_{i j k}\right\} ; k=1 ; 2 ; \ldots ; n$ was calculated according to expression (31) where $r=5$ was used.

\begin{tabular}{lcccccc}
\hline PCC (DL) & \multicolumn{3}{c}{ RMSE $(\mathrm{mm})$} & DIE (DL) & \multicolumn{3}{c}{ AD (mm) } \\
& Parabolic & Gaussian & Mean & & Parabolic & Gaussian \\
\hline Parabolic & $\backslash$ & 0.00592 & 0.49098 & Parabolic & $\backslash$ & 0.00477 \\
Gaussian & 1.00000 & $\backslash$ & 0.48993 & Gaussian & 0.00002 & $\backslash$ \\
Mean & 0.99377 & 0.99379 & $\backslash$ & Mean & 0.00309 & 0.00468 \\
\hline
\end{tabular}

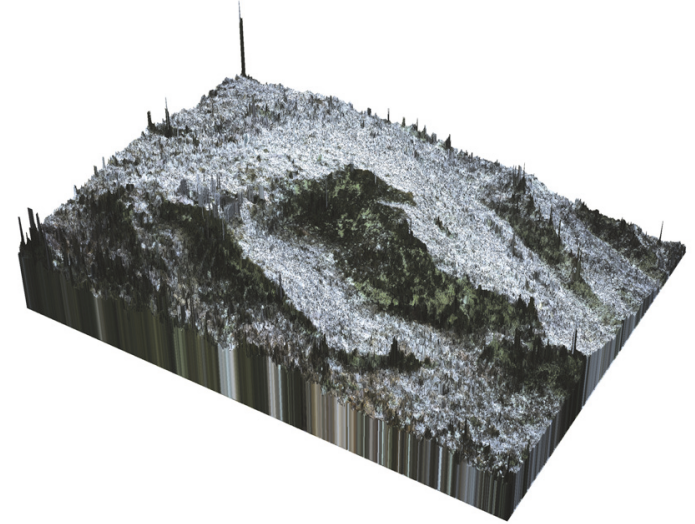

FIGURE 8: 3D reconstruction of the data from Figure 1: preprocessing according to Section 2.5 .1 (new method), the first step according to Section 2.5.2 (new method), and the second and the third steps according to Section 2.2 (present method). Expression (28) with $s=16$ was used in calculation of the matrix of sharpness detectors, its columns $\left\{{ }_{c} C_{i j k}\right\} ; k=1 ; 2 ; \ldots ; n$ was interpolated by the parabolic interpolation (present method).

Results illustrated in Figures 8 and 9 have been obtained by combination of present and proposed method (they are not realized by present methods only due to different scaling of the input data). Visualization of the parabolic interpolation of ${ }_{c} C$ is shown in Figure 8; Gaussian interpolation is illustrated in Figure 9. Practically the same and large noise is evident in these reconstructions.

Low-pass filters are commonly used to reduce the noise, but these filters are not ableto differentiate whether highfrequency information is caused by noise or by small details of useful information. Therefore, the loss of useful information is inevitable as shown in Figure 10.

In Figure 11, there is illustrated significantly better result of proposed method applied to the same matrix ${ }_{c} C$. There is no visible noise at the output, and high-frequency useful information (small surface details) is retained.

\section{Conclusion}

Reconstructions of three-dimensional object surfaces are an important task in many branches of research. Even though the standard method of imaging object surfaces is the use of confocal microscopes or laser scanners, there exist sophisticated mathematical methods that are able to process

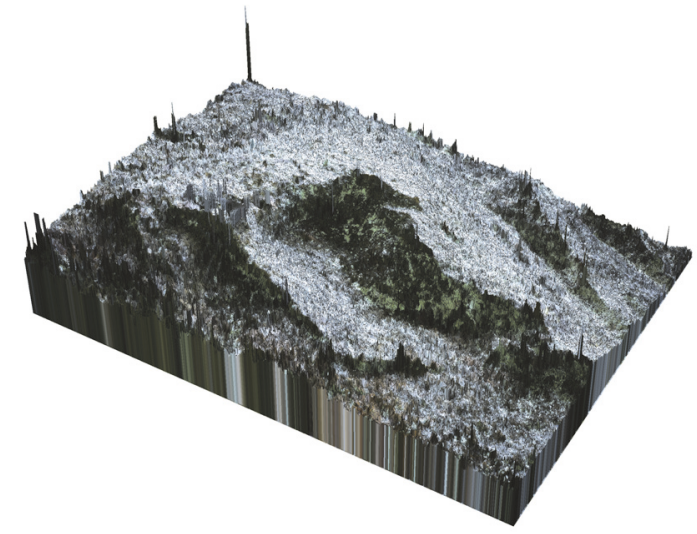

FIGURE 9: 3D reconstruction of the data from Figure 1: preprocessing according to Section 2.5.1 (new method), the first step according to Section 2.5.2 (new method), and the second and the third steps according to Section 2.2 (present method). Expression (28) with $s=16$ was used in calculation of the matrix of sharpness detectors, its columns $\left\{{ }_{c} C_{i j k}\right\} ; k=1 ; 2 ; \ldots ; n$ was interpolated by the Gaussian interpolation (present method).

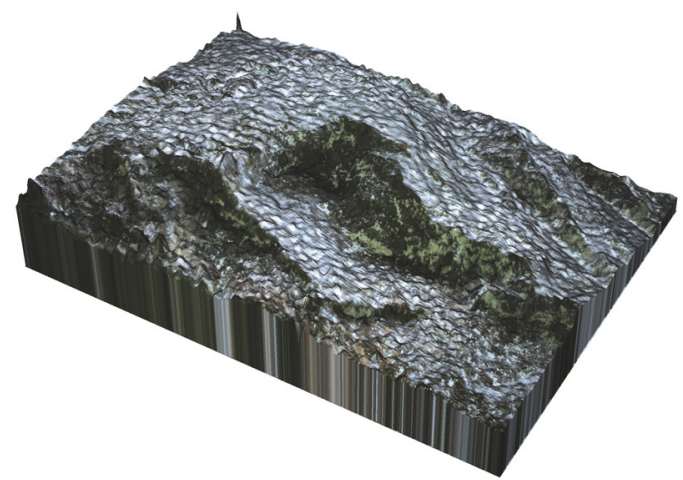

Figure 10: Gaussian low-pass filter with dispersion $\sigma^{2}=5$ applied to noisily output data from Figure 9. The noise is indeed reduced (but still visible); however useful high-frequency information is already lost.

images acquired by classic cameras and to construct $3 \mathrm{D}$ reconstruction similar to these from confocal microscopes or laser scanners. This enables us to obtain similar results with substantially less expensive equipment. 


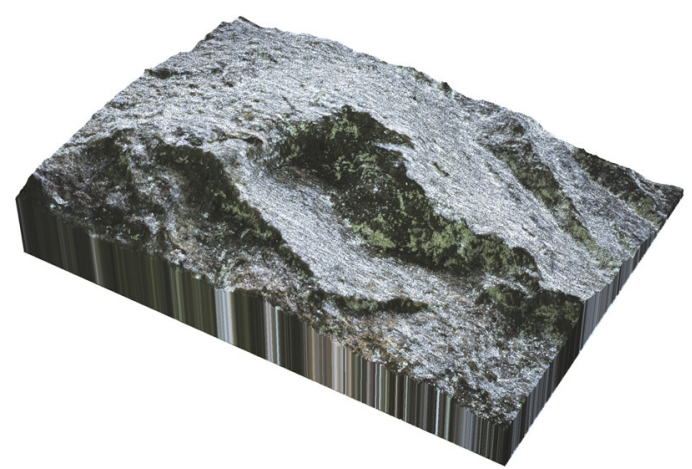

Figure 11: Completely new 3D reconstruction of the data from Figure 1: preprocessing according to Section 2.5.1, the first step according to Section 2.5.2-expression (28) with $s=16$ was usedand the second step according to Section 2.5.3, the expected values of its columns $\left\{{ }_{c} C_{i j k}\right\} ; k=1 ; 2 ; \ldots ; n$ was calculated according to expression (29) where $r=5$ was used.

\section{Conflicts of Interest}

The author declares that they have no conflicts of interest.

\section{Acknowledgments}

This work was supported by the Ministry of Education of the Czech Republic, Project LO1202 with financial subsidy, under the NPU I Programme. Thanks are due to Pavel Štarha for providing data.

\section{References}

[1] J. Halling, Introduction to tribology, Wykeham Books, London, UK, 1976.

[2] J. Bennett and L. Matton, Introduction to surface roughnessand scattering, Optical Society of America, Washington, DC, USA, 1999.

[3] W. Bowen and N. Hilal, Atomic force microscopy in proces engineering, Butterworth-Heinemann, Oxford, UK, 2009.

[4] S. Tolansky, "A light-profile microscope for surface studies," Zeitschrif tfur Elektrochemie, vol. 56, pp. 263-267, 1952.

[5] V. Thiery and D. I. Green, "The multifocus imaging technique in petrology," Computers \& Geosciences, vol. 45, pp. 131-138, 2012.

[6] P. de Groot, "Principles of interference microscopy for the measurement of surface topography," Advances in Optics and Photonics, vol. 7, no. 1, pp. 1-65, 2015.

[7] G. H. Kaufmann, Advances in speckle metrology and related techniques, Weinheim: Wiley, 2011.

[8] M. Mettanen and U. Hirn, "A comparison of five optical surface topography measurement methods," TAPPI Journal, vol. 14, no. 1, pp. 27-38, 2015.

[9] S. Bertin, H. Friedrich, P. Delmas, E. Chan, and G. Gimel'farb, "Digital stereo photogrammetry for grain-scale monitoring of fluvial surfaces: error evaluation and workflow optimisation," ISPRS Journal of Photogrammetry and Remote Sensing, vol. 101, pp. 193-208, 2015.
[10] S. Tang, X. Zhang, and D. Tu, "Micro-phase measuring profilometry: its sensitivity analysis and phase unwrapping," Optics and Lasers in Engineering, vol. 72, pp. 47-57, 2015.

[11] Q. Feng, "Novel methodsfor 3-D semi-automatic mapping of fracture geometry at exposed rock surfaces," KTH Dokotorsavhandling, 2001.

[12] S. Slob, B. Van Knapen, R. Hack, K. Turner, and J. Kemeny, "Method for automated discontinuity analysis of rock slopes with three-dimensional laser scanning," Transportation Research Record, no. 1913, pp. 187-194, 2005.

[13] S. Slob and R. Hack, "3D terrestrial laser scanning as a new field measurement and monitoring technique," in Engineering Geology for Infrastructure Planning in Europe, R. Hack, R. Azzam, and R. Charlier, Eds., pp. 179-189, Springer Verlag, Berlin, Heidelberg, Germany, 2004.

[14] P. Pawlus, M. Wieczorowski, and T. Mathia, The errors of stylus methods in Surface topography measurements, ZAPOL, Szczecin, Poland, 2014.

[15] J. Hoła, Ł. Sadowski, J. Reiner, and S. Stach, "Usefulness of 3D surface roughness parameters for nondestructive evaluation of pull-off adhesion of concrete layers," Construction and Building Materials, vol. 84, pp. 111-120, 2015.

[16] D. A. Agard, Z. Hiraoka, P. Shaw, and J. Sedat, "Flourescence microscopy in three dimensions," in Methods in Cell Biology, Part B: Quantitative flourescence microcopy-imaging and spectroscopy, vol. 30, pp. 359-362, San Diego: AcademicPress, California, CA, USA, 1989.

[17] J. B. Pawley, Handbook of Biological Confocal Microscopy, Springer US, Boston, MA, 1995.

[18] J. Pawley, Handbook of Confocal Microscopy, Plenum Press, New York, NY, USA, 1990.

[19] N. Logali, Confocal laser microscopy - principles, applicationsin medicine, biology, and the food sciences, Rijeka, Croatia, 2013.

[20] D. A. Lange, H. M. Jennings, and S. P. Shah, "Analysis of surface roughness using confocal microscopy," Journal of Materials Science, vol. 28, no. 14, pp. 3879-3884, 1993.

[21] Y. Ichikawa and J. Toriwaki, "Confocal microscope 3D visualizing method for fine surface characterization of microstructures," in Proceedings of SPIE 2862, Flatness, Roughness, and Discrete Defect Characterization for Computer Disks, Wafers, and Flat Panel Displays, pp. 96-101, Denver, Colo, USA.

[22] K. Nadolny, "Confocal laser scanning microscopy for characterisation of surface microdiscontinuities of vitrified bonded abrasive tools," International Journal of Mechanical Engineering and Robotics Research, vol. 1, pp. 14-29, 1.

[23] J. F. Brenner, B. S. Dew, J. B. Horton, T. King, P. W. Neurath, and W. D. Selles, "An automated microscope for cytologic research a preliminary evaluation," Journal of Histochemistry \& Cytochemistry, vol. 24, no. 1, pp. 100-111, 2017.

[24] R. J. Pieper and A. Korpel, "Image processing for extended depth of field," Applied Optics, vol. 22, no. 10, pp. 1449-1453, 1983.

[25] S. A. Sugimoto and Y. Ichioka, "Digital composition of images with increased depth of focus considering depth information," Applied Optics, vol. 24, no. 14, pp. 2076-2080, 1985.

[26] E. Krotkov, "Focusing," International Journal of Computer Vision, vol. 1, no. 3, pp. 223-237, 1988.

[27] J. Gillespie and R. A. King, "The use of self-entropy as a focus measure in digital holography," Pattern Recognition Letters, vol. 9, no. 1, pp. 19-25, 1989. 
[28] D. Agard, A. Hiraoka, Z. Shaw, and P. Sedat, "Flourescence microscopy in three dimensions," in Methods in Cell Biology, Part B: Quantitative flourescence microcopy-imaging and spectroscopy, D. L. Taylor and Y. Wang, Eds., vol. 30, pp. 359-362, Academic Press, San Diego, USA, 1989.

[29] D. Martišek, "The two-dimensional and three-dimensional processing of images provided by conventional microscopes," Scanning, vol. 24, no. 6, pp. 284-295, 2002.

[30] D. Martišek and H. Druckmüllerová, "Multifocal image processing," Mathematics for Applications, vol. 3, no. 1, pp. 77-90, 2014.

[31] M. Niederost, J. Niederost, and J. Ščučka, "International Archives of the Photogrammetry, Remote Sensing and Spatial Information Sciences XXXIV-5/W10," in Proceedings of the International Workshop on Visualization and Animation of Reality-based 3D Models, Tarasp-Vulpera, Switzerland, 2003.

[32] C. Kammerud, B. Abidi, S. Huq, and M. Abidi, "3D nanovision for the inspection of micro-electro-mechanical systems," in Proceedings of the 12th IEEE International Conference on Electronics, Circuits and Systems (ICECS 2005), Tunisia, December 2005.

[33] A. Thelen, S. Frey, S. Hirsch, and P. Hering, "Improvements in shape-from-focus for holographic reconstructions with regard to focus operators, neighborhood-size, and height value interpolation," IEEE Transactions on Image Processing, vol. 18, no. 1, pp. 151-157, 2009.

[34] S. K. Nayar and Y. Nakagawa, "Shape from Focus," IEEE Transactions on Pattern Analysis and Machine Intelligence, vol. 16, no. 8, pp. 824-831, 1994.

[35] T. Ficker and D. Martišek, "Three-dimensional reconstructions of solid surfaces using conventional microscopes," Scanning, vol. 38, no. 1, pp. 21-35, 2016.

[36] A. Thelen, Optimized surface extraction from Holographic data, Fakultat der Heinrch-Heine-Universitat Dusseldorf, Germany, 2006.

[37] D. Martišek, J. Procházková, and T. Ficker, "High-quality threedimensional reconstruction and noise reduction of multifocal images from oversized samples," Journal of Electronic Imaging, vol. 24, no. 5, Article ID 053029, 2015.

[38] H. Druckmüllerová, Phase-correlation based image registration, Brno University of technology, Czech Republic, 2010.

[39] W. K. Pratt, Digital Image Processing: PIKS Inside, John Wiley \& Sons, Inc., New York, USA, 3rd edition, 2001.

[40] B. Zitova and J. Flusser, "Image registration methods: a survey," Image and Vision Computing, vol. 21, no. 11, pp. 977-1000, 2003.

[41] M. V. Wyawahare et al., "Image registration techniques: an overview," International Journal of Signal Processing, Image Processing and Pattern Recognition, vol. 2, no. 3, 2009.

[42] E. M. Stern et al., Introduction to Fourier analysis on Euclidean spaces, Princeton, N. J. Princeton University Press, 1975. 


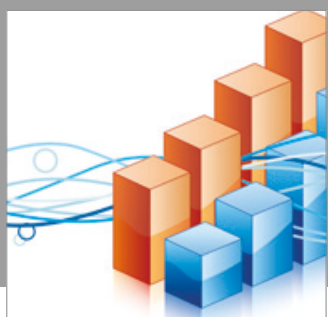

Advances in

Operations Research

vatersals

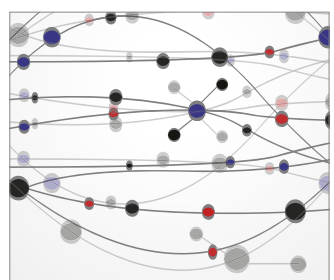

\section{The Scientific} World Journal
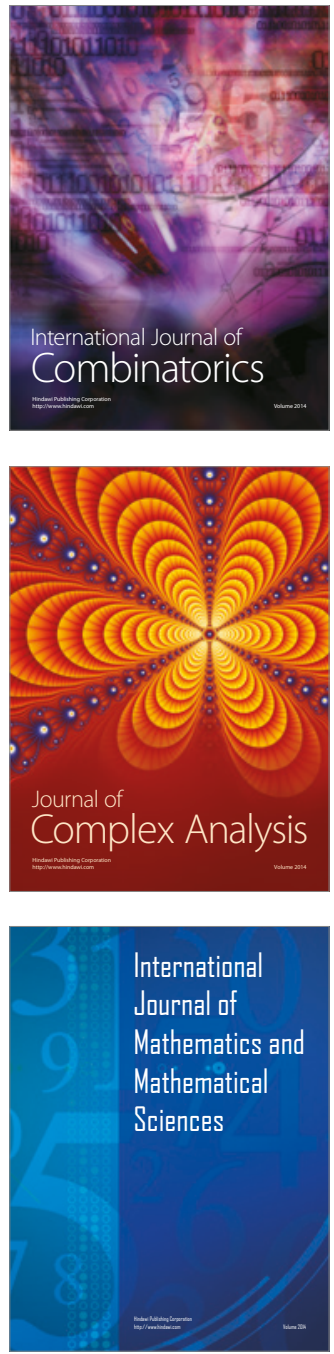
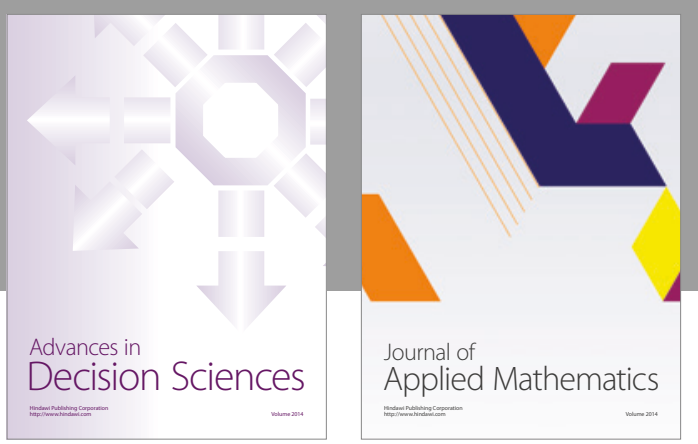

Algebra

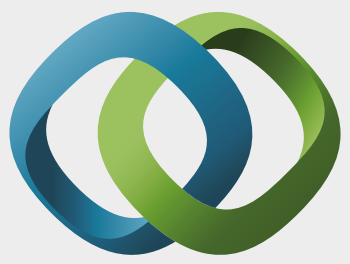

\section{Hindawi}

Submit your manuscripts at

https://www.hindawi.com
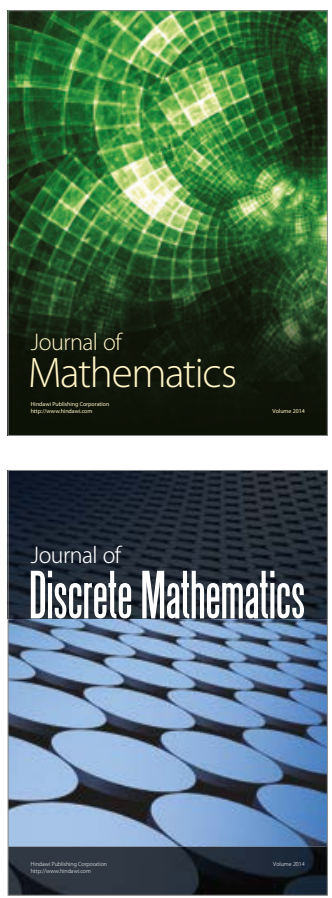

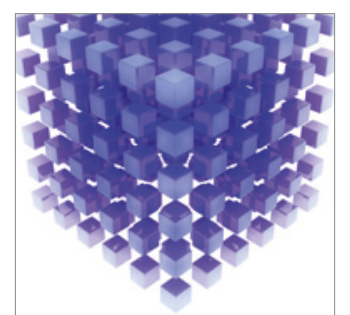

Mathematical Problems in Engineering
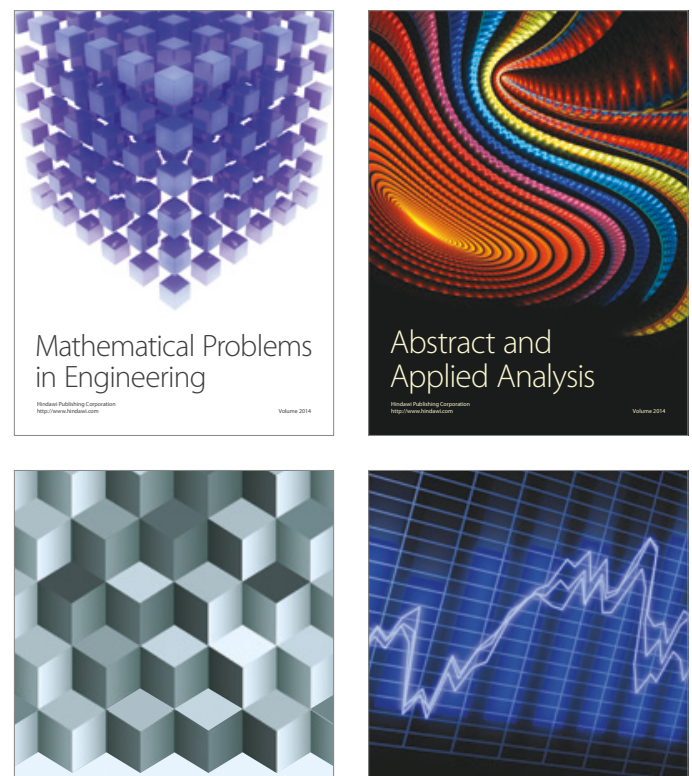

Journal of

Function Spaces

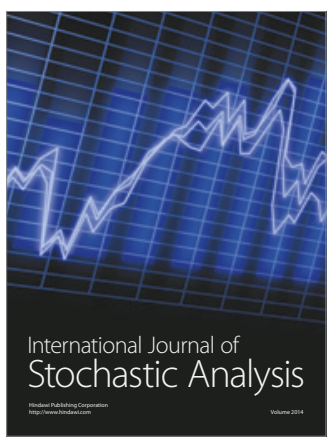

Probability and Statistics
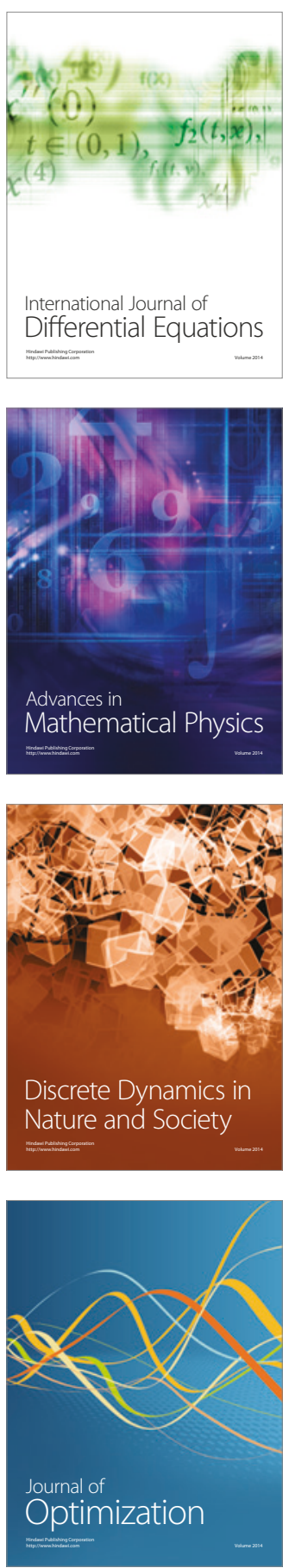